\title{
Magneto-optical probe of the fully gapped Dirac band in $\mathrm{ZrSiS}$
}

\author{
E. Uykur $\odot,{ }^{1, *}$ L. Z. Maulana $\odot,{ }^{1}$ L. M. Schoop, ${ }^{2}$ B. V. Lotsch, ${ }^{3,4}$ M. Dressel, ${ }^{1}$ and A. V. Pronin ${ }^{1}$ \\ ${ }^{1}$ 1. Physikalisches Institut, Universität Stuttgart, 70569 Stuttgart, Germany \\ ${ }^{2}$ Department of Chemistry, Princeton University, Princeton, New Jersey 08544, USA \\ ${ }^{3}$ Max Planck Institute for Solid State Research, 70569 Stuttgart, Germany \\ ${ }^{4}$ Chemistry Department, University of Munich (LMU), 81377 München, Germany
}

(Received 17 July 2019; published 6 November 2019)

\begin{abstract}
We present a far-infrared magneto-optical study of the gapped nodal-line semimetal $\mathrm{ZrSiS}$ in magnetic fields $B$ up to $7 \mathrm{~T}$. The observed field-dependent features, which represent intra- (cyclotron resonance) and interband transitions, develop as $\sqrt{B}$ in increasing field and can be consistently explained within a simple two-dimensional Dirac band model with a gap of $26 \mathrm{meV}$ and an averaged Fermi velocity of $3 \times 10^{5} \mathrm{~m} / \mathrm{s}$. This indicates a rather narrow distribution of these parameters along the in-plane portions of the nodal line in the Brillouin zone. A field-induced feature with an energy position that does not depend on $B$ is also detected in the spectra. Possible origins of this feature are discussed.
\end{abstract}

DOI: 10.1103/PhysRevResearch.1.032015

Three-dimensional nodal-line semimetals (NLSMs) [1] are currently a subject of intensive experimental investigations [2-6]. In these systems, two-dimensional (2D) electronic bands possessing linear dispersion cross each other along continuous lines in reciprocal space, hence enabling 2D Dirac electrons to exist in the 3D bulk. Among these materials, $\mathrm{ZrSiS}$ is probably the best studied system [7-14]. This material possesses two types of nodal lines: One of them is situated far away $(>0.5 \mathrm{eV})$ from the Fermi level $E_{F}$, while the second one, most interesting, is much closer to $E_{F}$ and is fully gapped due to spin-orbit coupling. This gap is calculated to be very small, of the order of $10 \mathrm{meV}$ [7]. Angle-resolved photoemission spectroscopy (ARPES) provides values between 15 and $50 \mathrm{meV}$ [8], while our earlier optical studies estimate the maximum of the gap to be around $30 \mathrm{meV}$ [14]. Although the low-energy nodal line in $\mathrm{ZrSiS}$ is slightly gapped, the linearity of the electronic bands forming this line extends up to $\sim 0.5 \mathrm{eV}$, and other nonlinear bands do not cross $E_{F}$. This makes $\mathrm{ZrSiS}$ one of the best systems to study the properties of Dirac electrons in NLSMs.

Magneto-optical spectroscopy is a powerful tool to investigate electronic properties of nodal semimetals and narrowband-gap materials [15-19]. For example, this method enables experimental verification of the electronic band structure by tracing the optical transitions between the magnetic-fieldinduced Landau levels (LLs). This approach is particularly relevant at low energies, where other experimental techniques often lack accuracy and resolution. Here, we report on far-infrared magneto-optical investigations of $\mathrm{ZrSiS}$. We

\footnotetext{
*ece.uykur@pi1.physik.uni-stuttgart.de
}

Published by the American Physical Society under the terms of the Creative Commons Attribution 4.0 International license. Further distribution of this work must maintain attribution to the author(s) and the published article's title, journal citation, and DOI. found that Dirac quasiparticles fully dominate the ac (magneto)transport in $\mathrm{ZrSiS}$ : Both inter- and intraband optical transitions demonstrate a square-root dependence on the magnetic field, typical for such bands. All measured magneto-optical spectra can be well described by a simple model of gapped Dirac bands with a single (i.e., k-independent) gap of $26 \mathrm{meV}$.

The sample studied was grown by the method described elsewhere [7], and was the same single crystal previously used for the zero-field optical measurements of Ref. [14]. The optical reflectivity spectra were collected from (001) surfaces (the in-plane response) utilizing a home-build magnetooptical setup connected to a commercial Fourier-transform infrared spectrometer. The spectra were recorded for photon energies between $\sim 5$ and $75 \mathrm{meV}$ in magnetic fields up to $7 \mathrm{~T}$ at $10 \mathrm{~K}$. The Voigt geometry was chosen as the measurement configuration to allow the Kramers-Kronig analysis for linearly polarized light [20] (in the commonly utilized Faraday geometry, such an analysis would only be meaningful for circular polarizations, which cannot be used for broadband optical measurements). The spectra have been obtained with two linear polarizations, $\tilde{\mathbf{E}} \| \mathbf{B}$ and $\tilde{\mathbf{E}} \perp \mathbf{B}$ (here, $\tilde{\mathbf{E}}$ is the electric component of the probing radiation and $\mathbf{B}$ is the external magnetic field). Hereafter we describe our results obtained for $\tilde{\mathbf{E}} \perp \mathbf{B}$, while in the $\tilde{\mathbf{E}} \| \mathbf{B}$ polarization no fieldinduced changes were detected. A simultaneous change of the $\mathbf{B}$-field direction and the light polarization (keeping the angle between them fixed) with respect to the crystallographic direction within the (001) plane did not affect the spectra due to the tetragonal crystal symmetry within this plane. In order to obtain the optical conductivity, the measured reflectivity spectra were merged with the zero-field reflectivity at higher energies (up to $\sim 5.6 \mathrm{eV}$, [14]) and the Kramers-Kronig analysis was performed using $\mathrm{x}$-ray atomic scattering functions [21] and Drude-Lorentz fits as extrapolations at high and low frequencies, respectively.

The measured reflectivity is given in Fig. 1. To demonstrate the $B$-field-induced changes more clearly, we plot the relative 

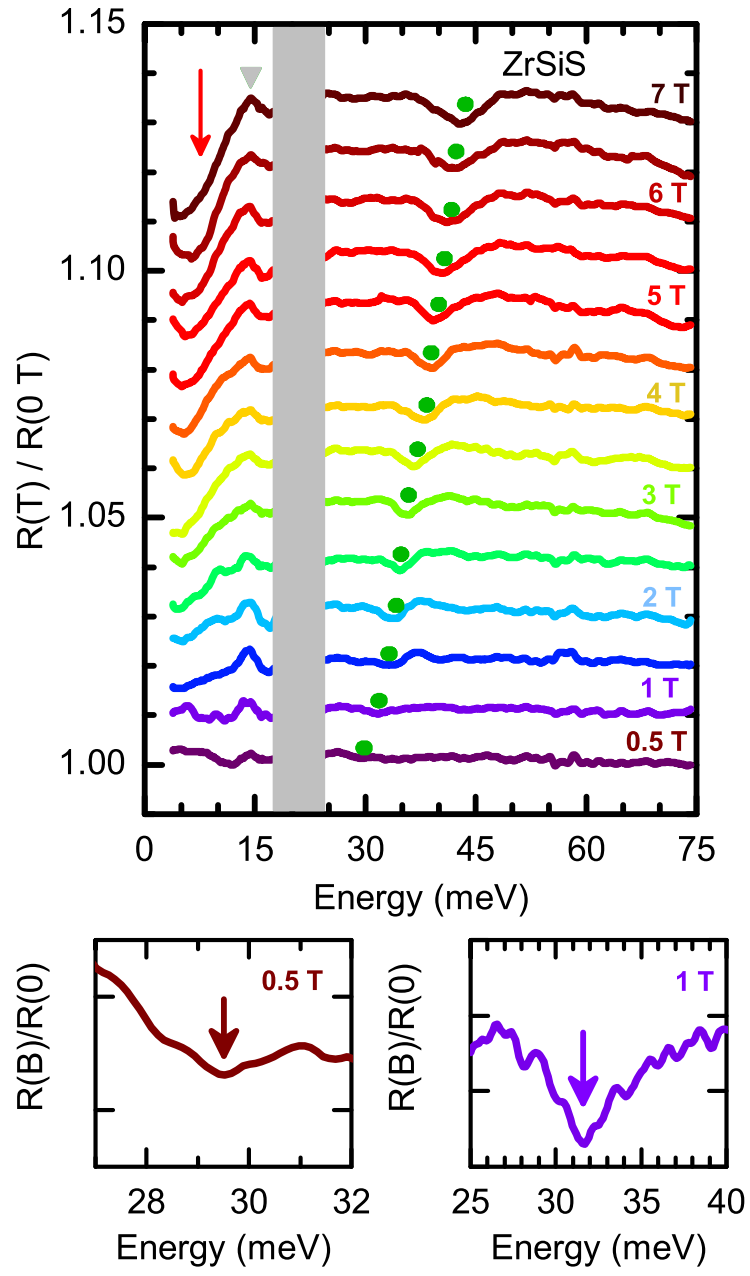

FIG. 1. Magnetoreflectance spectra of $\mathrm{ZrSiS}$ normalized to zerofield reflectivity. The spectra at various fields are shifted by 0.01 with respect to the previous one for clarity. The shaded area is the range not accessible for our measurement setup. Enlarged spectra near one of the absorption modes for 0.5 and $1 \mathrm{~T}$ are given in the bottom panels.

reflectivity, $R(B) / R(B=0)$. The corresponding spectra of the real part of optical conductivity, normalized by its zero-field value, $\sigma(B) / \sigma(B=0)$, are given in Fig. 3(a). The spectra between $\sim 17$ and $24 \mathrm{meV}$ are largely affected by noise in our setup and therefore eliminated from the discussion. Above $50 \mathrm{meV}$ no field-induced features appear; we chose the energy scale on the plots accordingly.

The optical spectra demonstrate three field-induced features: (i) a suppression of reflectivity and optical conductivity at the lowest frequencies (marked with the red arrows in both figures); (ii) a feature that grows in intensity with increasing $B$, but remains its frequency position constant at around $15 \mathrm{meV}$ [the gray triangle in Fig. 1 and the strongest peak in Fig. 3(a)]; and finally, (iii) a feature around $30-45 \mathrm{meV}$ seen at all the fields and marked with the green circles in Fig. 1 and the green arrow in Fig. 3(a) demonstrating the frequency shift of the feature as $B$ increases. In Fig. 3(b), a false-color plot of the relative optical conductivity as a function of energy and magnetic field is shown. The three field-induced features are seen in this plot as changes of color. Features (i) and (iii) are further emphasized by the open symbols obtained as discussed below.

Before going to the discussion, let us recall that for gapped Dirac bands the LL spectrum reads as [18,22]

$$
E_{ \pm n}= \pm \sqrt{2 e \hbar|n| B v_{F}^{2}+\left(\frac{\Delta}{2}\right)^{2}},
$$

where $n$ is the LL index, $v_{F}$ is the (average) Fermi velocity, and $\Delta$ is the gap between conduction and valence bands. The LLs in the conduction and valence bands correspond to the "+" and "-" signs in Eq. (1), respectively. The spectrum of Eq. (1) is shown in the upper panel of Fig. 4. For the allowed inter-LL transitions, $|n|$ can be changed by \pm 1 . Hence, the transition energy can be written, e.g., as

$E_{t}=\sqrt{2 e \hbar(|n|+1) B v_{F}^{2}+\left(\frac{\Delta}{2}\right)^{2}} \pm \sqrt{2 e \hbar|n| B v_{F}^{2}+\left(\frac{\Delta}{2}\right)^{2}}$,

with the plus sign corresponding to interband and the minus sign to intraband transitions. These transitions are schematically depicted in the bottom part of Fig. 4. Two situations are considered: with $E_{F}$ in the gap (left panel) and in the conduction band (right panel). Both situations are relevant for $\mathrm{ZrSiS}$ : It is well known that the Fermi level crosses electronic bands along some portions of the nodal line, while it is within the gap elsewhere [7]. In the following, we argue that this simple model can account for all observed features, which frequency position changes as a function of applied field.

It is worth noting here that optical conductivity measurements are not $k$ sensitive and probe the entire Brillouin zone. In the present study, we mostly probe the in-plane parts of the nodal line, because the external magnetic field is applied parallel to the (001) plane and hence the closed cyclotron orbits are formed for the carriers from the bands forming the in-plane portions of the nodal line (see Fig. 2). (Small contributions from the out-of-plane parts can also be present due to their corrugations.)

We start from the lowest-energy feature (i). To make it more visible, we added the red open circles to Fig. 3(b). Their positions are determined as the unity-crossing points of $\sigma(B) / \sigma(B=0)$ [cf. Fig. 3(a)]. Feature (i) starts to appear at $1.5 \mathrm{~T}$ and seems to extrapolate to zero as $B \rightarrow 0$. By looking on the field evolution of this feature in Fig. 3(a), it can clearly be related to depletion of the Drude response: The loss of Drude spectral weight at low energies is expected to be transferred to a cyclotron-resonance (CR) mode [16], as indicated by the dashed-dotted pink arrow in Fig. 3(a). In our measurements the cyclotron resonance is superimposed on the field-independent feature (ii). In order to extract the CR parameters, we performed a Drude-Lorentz fit [23] that takes into account the CR itself, the leftover Drude component, the field-independent resonance, and the high-energy mode corresponding to feature (iii), the latter being taken into account only for completeness. In Fig. 3(c), we present an example of such a fit for $1.5 \mathrm{~T}$. This way we can extract the field dependence of the cyclotron resonance. In the inset of the Fig. 3(c) we plot the obtained energies of the cyclotron resonance as a function of the $B$ field. The linear behavior on 


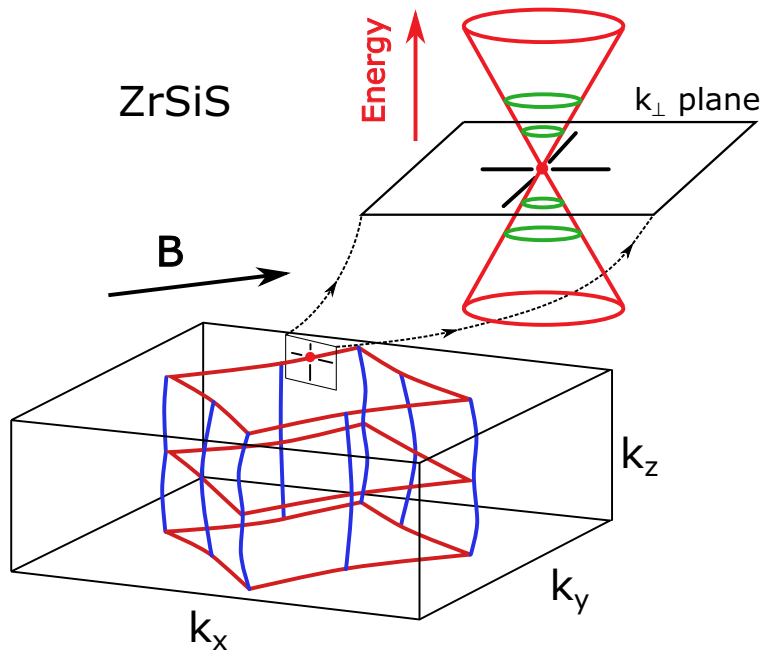

FIG. 2. Schematics of the nodal line in ZrSiS. The 2D Dirac band with its Landau levels formed for an in-plane orientation of $\mathbf{B}$ is sketched in the upper right corner $\left(k_{\perp}\right.$ stands for the $k$ directions perpendicular to the nodal line). The band gap is not shown for simplicity.

the $\sqrt{B}$ scale proves the relativistic nature (linear bands) of the carriers responsible for this resonance and can be nicely fitted by Eq. (2) using $n=0$ and the minus sign corresponding to the intraband transition (the red arrow in Fig. 4).
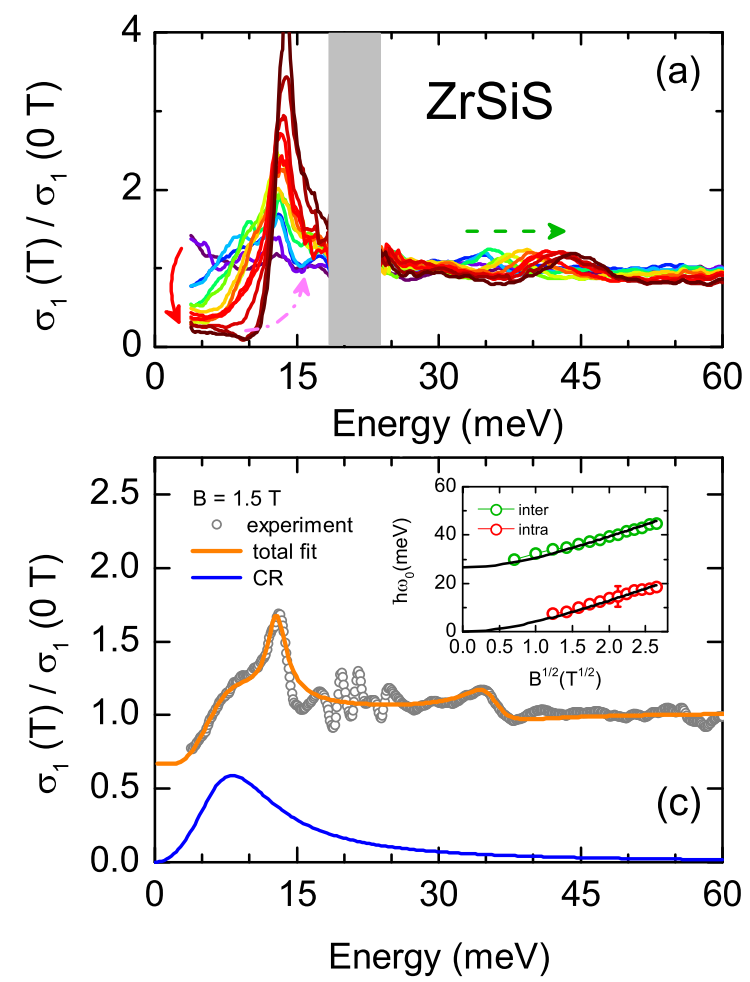

Now, we turn to feature (iii). At low $B$, it extrapolates to the gap detected in our zero-field measurements [14] and it can be assigned to the transitions between LLs in the valence and conduction bands. In Fig. 3(b), we plot the peak positions of $\sigma(B) / \sigma(B=0)$ as open green symbols. Remarkably, we can fit the symbols with Eq. (2) using the same $\Delta$ and $v_{F}$ as we used to fit the CR mode. Only the sign between the addends should be changed (minus to plus). Thus, the observed mode (iii) corresponds to the interband transitions involving the $n=0$ LLs, i.e., the transitions from $n=-1$ to $n=+0$ or from $n=-0$ to $n=+1$ (green arrows in Fig. 4).

The fits to the $B$-dependent modes (i) and (iii) shown in Figs. 3(b) and 3(c) as black solid lines are all performed using the single values of $\Delta=26 \mathrm{meV}$ and $v_{F}=3 \times 10^{5} \mathrm{~m} / \mathrm{s}$. This strongly indicates that the distribution of these parameters along (the in-plane portions of) the nodal line in the Brillouin zone is rather narrow, as optical probes may only provide momentum-averaged quantities. Had $\Delta$ and $v_{F}$ possessed strong $\mathbf{k}$ dispersions, the field-induced magneto-optical features would be extremely broad, if detectable. As already noticed, the gap identified is well within the margins provided by calculations [7], ARPES [8], and optics [14]. The same is true for the obtained Fermi velocity (cf. Refs. $[10,24]$ ).

In Fig. 3(b), we also show the position expected for the inter-LL transitions from $n=-2$ to $n=+1$ and from $n=-1$ to $n=+2$ (dotted line, see also the dotted arrows in Fig. 4). These, as well as other transitions between the LLs with larger $n$, are not detectable in our spectra. This situation is quite

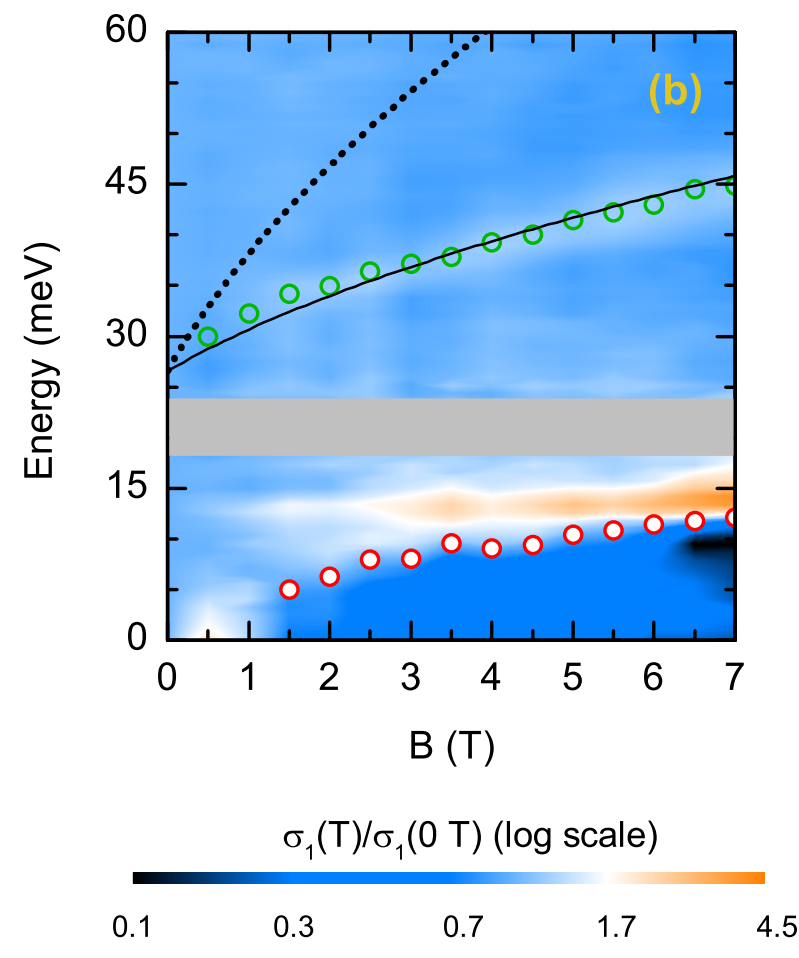

FIG. 3. (a) Relative optical conductivity of ZrSiS vs frequency for all studied fields. (b) Same data as a false-color plot. Lines and dots correspond to different inter-LL transitions as discussed in the text. (c) An example of the Lorentz-Drude fit to the optical conductivity data at $1.5 \mathrm{~T}$. The structures between approximately 17 and $24 \mathrm{meV}$ are noise (corresponding to the shaded areas in the other panels). The inset shows the energy positions $\left(\hbar \omega_{0}\right)$ of the inter- and intraband transitions determined from the conductivity data and fitted with Eq. (2). Note the $\sqrt{B}$ horizontal scale. 

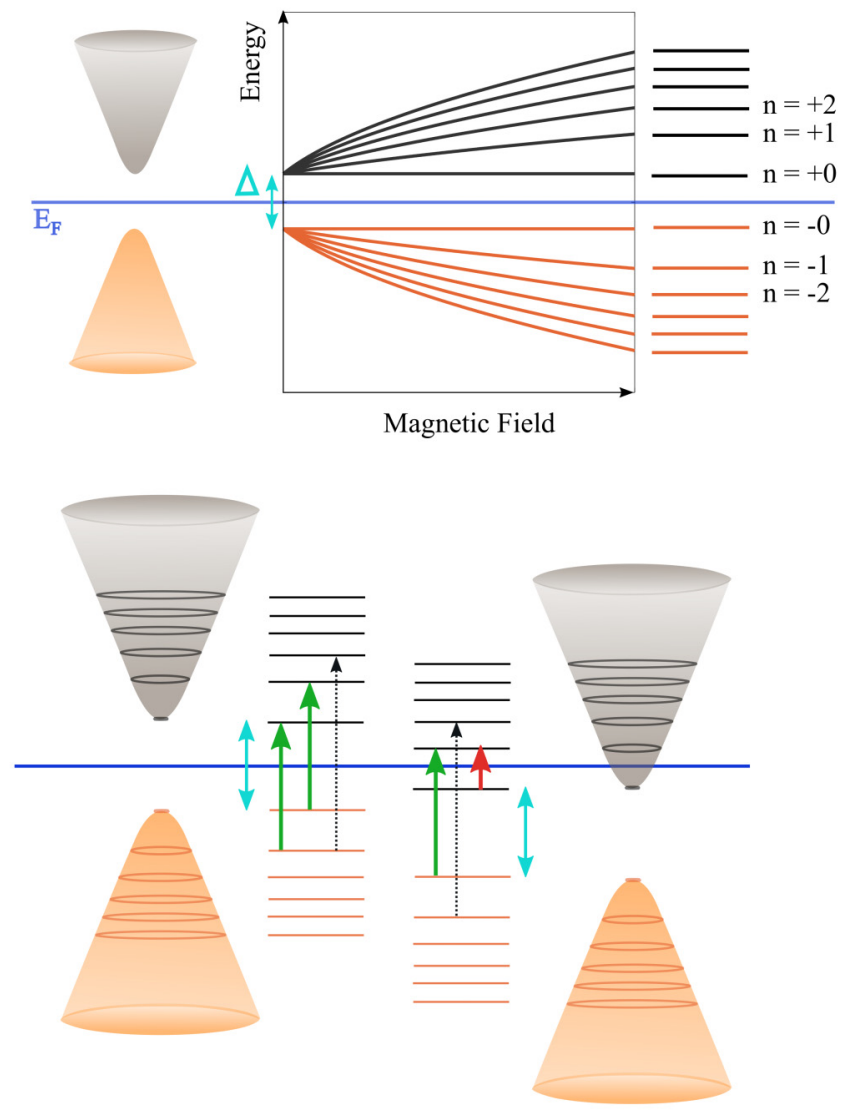

FIG. 4. Upper panel: Schematic representation of Landau levels appearing in a gapped Dirac band. Bottom panels sketch the 2D Dirac band in $\mathrm{ZrSiS}$. Two cuts perpendicular to the nodal line are shown: Along some portions of the gapped nodal line, the Fermi level is in the gap (left picture), while in the other portions (right picture) it is in the conduction band. The cyan solid arrow represents the determined single band gap. Other solid arrows depict the observed transitions: The intraband (CR) transition $+0 \rightarrow+1$ is shown as a red arrow, while the interband transitions $(-0 \rightarrow+1$ and $-1 \rightarrow+0)$ are given as green arrows. The dotted arrows show other possible interband transitions, which are not observed in this work. The color code for the arrows is consistent with the one used to mark the transition modes in Figs. 1 and 3.

common: The line broadening increases with energy [15,25], making the line detection difficult.

Finally, let us turn to the field-independent feature (ii), which is seen around $15 \mathrm{meV}$. The position of this mode does not show any appreciable frequency shift, but the mode strength increases with magnetic field. A simple explanation of this mode would be an in-gap impurity resonance. However, the studied $\mathrm{ZrSiS}$ sample is very clean: The mean free path extracted from our optical measurements on this very sample is of the order of $1 \mu \mathrm{m}$ at low temperatures [14]. Thus, this explanation seems not to be very likely. Alternatively, surface states [26] might be relevant for the formation of this mode. A more appealing option is of bulk and intrinsic origin. We note that a low-energy mode with a very similar behavior in the magnetic field (constant energy position and strength increasing with $B$ ) was observed, but remained unexplained, in another gapped NLSM, NbAs 2 [19]. It would be tempting to assign both modes to the $B$-independent resonance, recently theoretically proposed to be a hallmark of the NLSM state [27]. However, the model used in this reference (a single nodal loop formed by crossing cones) is far too simple and not directly applicable to $\mathrm{ZrSiS}$ or $\mathrm{NbAs}_{2}$ : Both compounds possess complex and extended nodal lines. Thus, we call for more theoretical studies in this direction.

Summarizing, our in-plane far-infrared magneto-optical study of $\mathrm{ZrSiS}$ reveals three features developing in the applied magnetic field. The energy position of two of them scales as a square root of $B$. A simple model of a single gapped Dirac band provides an adequate explanation for the field evolution of these features, the low-energy feature being associated with intraband (cyclotron resonance) absorption, while the high-energy one appearing due to interband LL transitions. The free parameters of this model are defined as $\Delta=(26 \pm 2) \mathrm{meV}$ and $v_{F}=(3.0 \pm 0.2) \times 10^{5} \mathrm{~m} / \mathrm{s}$, which can be considered as averaged values over the entire in-plane part of the nodal line. Both values are well consistent with the ones obtained by other methods and reported in the literature. The fact that such a simple model accounts for the complete magneto-optical spectrum indicates that $\Delta$ and $v_{F}$ do not vary appreciably along (the in-plane portion of) the nodal line. This also evidences that other bands do not contribute to the corresponding ac transport. Possible origins of the third observed mode are discussed and more theory output is called for.

The authors acknowledge fruitful discussions with Chao Zhang (Wollongong), Milan Orlita (Grenoble), and Sascha Polatkan (Stuttgart) and the technical support by Gabriele Untereiner (Stuttgart). The work was supported by the Deutsche Forschungsgesellschaft (DFG) via DR228/51-1 and the Max Planck Society. E.U. acknowledges the support by the ESF and by the Ministry of Science, Research, and Arts of Baden-Württemberg. Work at Princeton was supported by NSF through the Princeton Center for Complex Materials, a Materials Research Science and Engineering Center (Grant No. DMR-1420541).
[1] A. A. Burkov, M. D. Hook, and L. Balents, Phys. Rev. B 84, 235126 (2011).

[2] C. Fang, H. Weng, X. Dai, and Z. Fang, Chin. Phys. B 25, 117106 (2016).

[3] G. Bian, T.-R. Chang, R. Sankar, S.-Y. Xu, H. Zheng, T. Neupert, C.-K. Chiu, S.-M. Huang, G. Chang, I.
Belopolski, D. S. Sanchez, M. Neupane, N. Alidoust, C. Liu, B. Wang, C.-C. Lee, H.-T. Jeng, C. Zhang, Z. Yuan, S. Jia et al., Nat. Commun. 7, 10556 (2016).

[4] R. Yu, H. Weng, Z. Fang, X. Dai, and X. Hu, Phys. Rev. Lett. 115, 036807 (2015). 
[5] Y. Chen, Y.-M. Lu, and H.-Y. Kee, Nat. Commun. 6, 6593 (2015).

[6] L. S. Xie, L. M. Schoop, E. M. Seibel, Q. D. Gibson, W. Xie, and R. J. Cava, APL Mater. 3, 083602 (2015).

[7] L. M. Schoop, M. N. Ali, C. Straßer, A. Topp, A. Varykhalov, D. Marchenko, V. Duppel, S. S. P. Parkin, B. V. Lotsch, and C. R. Ast, Nat. Commun. 7, 11696 (2016).

[8] C. Chen, X. Xu, J. Jiang, S.-C. Wu, Y. P. Qi, L. X. Yang, M. X. Wang, Y. Sun, N. B. M. Schröter, H. F. Yang, L. M. Schoop, Y. Y. Lv, J. Zhou, Y. B. Chen, S. H. Yao, M. H. Lu, Y. F. Chen, C. Felser, B. H. Yan, Z. K. Liu, and Y. L. Chen, Phys. Rev. B 95, 125126 (2017).

[9] M. Neupane, I. Belopolski, M. M. Hosen, D. S. Sanchez, R. Sankar, M. Szlawska, S.-Y. Xu, K. Dimitri, N. Dhakal, P. Maldonado, P. M. Oppeneer, D. Kaczorowski, F. Chou, M. Z. Hasan, and T. Durakiewicz, Phys. Rev. B 93, 201104(R) (2016).

[10] M. N. Ali, L. M. Schoop, C. Garg, J. M. Lippmann, E. Lara, B. Lotsch, and S. S. P. Parkin, Sci. Adv. 2, e1601742 (2016).

[11] S. Pezzini, M. R. van Delft, L. M. Schoop, B. V. Lotsch, A. Carrington, M. I. Katsnelson, N. Hussey, and S. Wiedmann, Nat. Phys. 14, 178 (2017).

[12] M. Matusiak, J. R. Cooper, and D. Kaczorowski, Nat. Commun. 8, 15219 (2017).

[13] J. Hu, Z. Tang, J. Liu, Y. Zhu, J. Wei, and Z. Mao, Phys. Rev. B 96, 045127 (2017).

[14] M. B. Schilling, L. M. Schoop, B. V. Lotsch, M. Dressel, and A. V. Pronin, Phys. Rev. Lett. 119, 187401 (2017).

[15] M. Orlita, C. Faugeras, R. Grill, A. Wysmolek, W. Strupinski, C. Berger, W. A. de Heer, G. Martinez, and M. Potemski, Phys. Rev. Lett. 107, 216603 (2011).
[16] M. Orlita, I. Crassee, C. Faugeras, A. B. Kuzmenko, F. Fromm, M. Ostler, Th. Seyller, G. Martinez, M. Polini, and M. Potemski, New. J. Phys. 14, 095008 (2012).

[17] Z.-G. Chen, R. Y. Chen, R. D. Zhong, J. Schneeloch, C. Zhang, Y. Huang, F. Qu, R. Yu, Q. Li, G. D. Gu, and N. L. Wang, Proc. Natl. Acad. Sci. USA 114, 816 (2017).

[18] Z.-G. Chen, Z. Shi, W. Yang, X. Lu, Y. Lai, H. Yan, F. Wang, G. Zhang, and Z. Li, Nat. Commun. 5, 4461 (2014).

[19] Y. Shao, Z. Sun, Y. Wang, C. Xu, R. Sankar, A. J. Breindel, C. Cao, M. M. Fogler, A. J. Millis, F. Chou, Z. Li, T. Timusk, M. B. Maple, and D. N. Basov, Proc. Natl. Acad. Sci. USA 116, 1168 (2019).

[20] E. D. Palik and J. K. Furdyna, Rep. Prog. Phys. 33, 1193 (1970).

[21] D. B. Tanner, Phys. Rev. B 91, 035123 (2015).

[22] X. Chen, J. R. Wallbank, A. A. Patel, M. Mucha-Kruczyński, E. McCann, and V. I. Fal'ko, Phys. Rev. B 89, 075401 (2014).

[23] M. Dressel and G. Grüner, Electrodynamics of Solids (Cambridge University Press, Cambridge, UK, 2002).

[24] M. S. Lodge, G. Chang, C.-Y. Huang, B. Singh, J. Hellerstedt, M. T. Edmonds, D. Kaczorowski, M. M. Hosen, M. Neupane, H. Lin, M. S. Fuhrer, B. Weber, and M. Ishigami, Nano Lett. 17, 7213 (2017).

[25] I. O. Nedoliuk, S. Hu, A. K. Geim, and A. B. Kuzmenko, Nat. Nanotechnol. 14, 756 (2019).

[26] A. Topp, R. Queiroz, A. Grüneis, L. Müchler, A. W. Rost, A. Varykhalov, D. Marchenko, M. Krivenkov, F. Rodolakis, J. L. McChesney, B. V. Lotsch, L. M. Schoop, and C. R. Ast, Phys. Rev. X 7, 041073 (2017).

[27] W. Duan, C. Yang, Z. Ma, Y. Zhu, and C. Zhang, Phys. Rev. B 99, 045124 (2019). 